\title{
A hybrid approach for the efficient synthesis of renewable energy systems
}

Article in Applied Energy · December 2014

Impact Factor: $5.61 \cdot$ DOI: 10.1016/j.apenergy.2014.03.051

CITATIONS

18

6 authors, including:

\section{Goran Gasparovic}

15 PUBLICATIONS 36 CITATIONS

SEE PROFILE

\section{Goran Krajacic}

University of Zagreb

67 PUBLICATIONS 465 CITATIONS

SEE PROFILE
Philip Voll

RWTH Aachen University

14 PUBLICATIONS 100 CITATIONS

SEE PROFILE

Neven Duic

University of Zagreb

409 PUBLICATIONS 1,637 CITATIONS

SEE PROFILE 


\title{
A hybrid approach for the efficient synthesis of renewable energy systems
}

\author{
Philipp Petruschke ${ }^{\mathrm{a}}$, Goran Gasparovic ${ }^{\mathrm{b}}$, Philip Voll ${ }^{\mathrm{a}}$, Goran Krajačić ${ }^{\mathrm{b}}$, Neven Duić ${ }^{\mathrm{b}}$, André Bardow ${ }^{\mathrm{a}, *}$ \\ a Institute of Technical Thermodynamics, RWTH Aachen University, Schinkelstr. 8, 52062 Aachen, Germany \\ ${ }^{\mathrm{b}}$ Department of Energy, Power Engineering and Environment, University of Zagreb, Ivana Lucica 5, HR-10000 Zagreb, Croatia
}

\section{H I G H L I G H T S}

- A hybrid synthesis method for renewable energy systems is presented.

- Heuristic equipment preselection is combined with superstructure-based optimization.

- A real world case study is analyzed (Mljet Island, Croatia).

- Investment cost savings of up to 59\% compared to classical simulation are achieved.

- The computational effort required for optimization is low.

\section{A R T I C L E I N F O}

\section{Article history:}

Received 2 December 2013

Received in revised form 4 March 2014

Accepted 21 March 2014

Available online 14 April 2014

\section{Keywords:}

Renewable energy systems

Synthesis

Optimization

Heuristics

RenewIslands

Superstructure

\begin{abstract}
A B S T R A C T
An efficient synthesis method for renewable energy systems is presented that exploits synergies between heuristic- and optimization-based approaches. For this purpose, the RenewIslands method has been integrated into a superstructure-based optimization approach. The resulting hybrid approach consists of two steps: First, heuristic-based equipment preselection identifies a set of promising candidate technologies. Next, the preselected technologies are employed in superstructure-based optimization to determine the optimal renewable energy system. The heuristic preselection systematically avoids excessively large superstructures, while the subsequent optimization ensures that the optimal solution is selected. The proposed method is applied to the case of Mljet Island, Croatia. A renewable energy system is synthesized that requires 59\% less investment costs compared to the solution derived by classical simulation. At the same time, the optimization problem is solved in less than $2 \mathrm{~min}$. The proposed hybrid method is shown to provide an efficient route to the synthesis of renewable energy systems.
\end{abstract}

๑) 2014 Elsevier Ltd. All rights reserved.

\section{Introduction}

The synthesis of energy supply systems with renewable resources is a key lever for facing the challenges of sustainable development and climate protection [1-3]. However, the synthesis problem is an inherently difficult task for which three hierarchically-dependent levels need to be taken into account [4] (Fig. 1): The configuration level where equipment choices are made, the sizing level where equipment's capacities are determined and the operational level where the actual load dispatch is specified. While these levels need to be considered for any energy system, a key challenge in the synthesis of renewable energy systems is to cope with the complexity stemming from the temporal and spatial interdependencies associated with renewable resources, usually

\footnotetext{
* Corresponding author. Tel.: +49 2418095380.

E-mail address: andre.bardow@ltt.rwth-aachen.de (A. Bardow).
}

requiring the installation of storage systems [5]. Additionally, the variety of available technologies and possible combinations adds significantly to the complexity. Besides, both the associated economic and ecological impacts have to be considered since the use of renewables is still usually motivated by environmental arguments. Thus, to find the best solution for a given synthesis problem, complex relationships and trade-offs between technical, economic and ecological consequences have to be balanced.

For the solution of such synthesis problems, two types of approaches are widely followed [4]: Traditionally, heuristic-based approaches are used, but also optimization-based approaches have been developed. Heuristic-based approaches typically rely on specific expert knowledge or physical insights to define possible energy systems and analyze them in simulation studies [6-12]. On the one hand, the heuristic-based approach is usually robust and generates solutions with manageable effort. On the other hand, only a limited number of alternatives can be studied in 


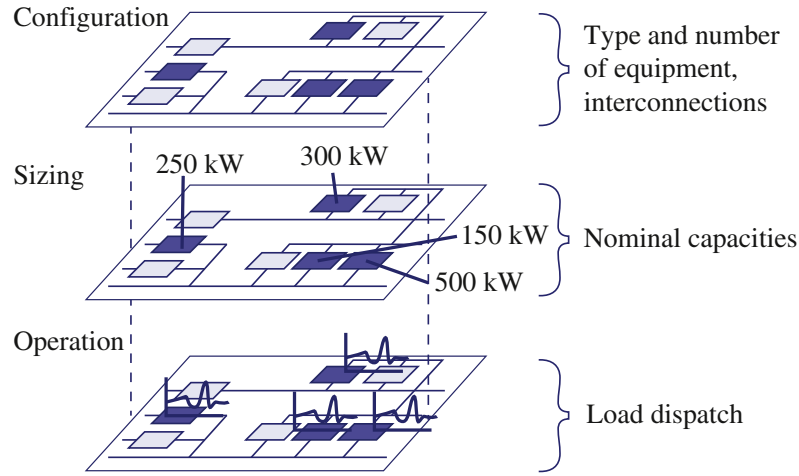

Fig. 1. Hierarchically-dependent levels configuration, sizing and operation to be taken into account for the synthesis of energy supply systems.

simulation studies and the risk to overlook superior solutions is high [4]. In contrast, optimization-based synthesis approaches allow for the investigation of a virtually unlimited number of alternatives and thus generally enable to find the optimal solution among all possible alternatives [13-20]. However, for large problems the modeling effort and solution times can become prohibitively large $[21,22]$.

To combine the advantages from heuristic and optimizationbased approaches, so called hybrid approaches have been successfully developed in other fields [23]. In this work, a hybrid approach is developed for the efficient synthesis of renewable energy systems. The proposed method builds upon the RenewIslands method by Duić et al. [24] and the automated superstructure-based optimization approach developed by Voll et al. [25].

This paper is organized as follows: In Section 2, the proposed hybrid approach is presented. In Section 3, a real world case study is considered - the island of Mljet, Croatia. The proposed method is applied to synthesize a $100 \%$ renewable energy system. The solution is compared to findings from an earlier publication where the RenewIslands method has been applied to the identical case but without rigorous optimization [26]. In Section 4, the benefits of the optimization-based synthesis are further elaborated and the required solution effort is analyzed. Finally, the paper is summarized (Section 5).

\section{A hybrid approach for the synthesis of renewable energy systems}

The proposed hybrid approach combines two well-founded synthesis methods. The RenewIslands method has been developed for energy planning of isolated islands $\underline{[27]}$ and has been implemented into the H2RES software $[11,28]$. Its core concept is to use heuristic rules to evaluate and structure information on local resources and demands, select promising renewable technologies and devise possible energy systems. The inputs are qualitative statements about the energy demand levels and the available resources which are classified as "low", "medium" or "high". A range of if-then-relations is then provided to derive a set of promising technologies. Based on this set of technologies, synthesis alternatives to be considered are heuristically defined by the user and assessed in scenario-type simulation studies (for details the reader is referred to [24]). The major strength of the RenewIslands method is that it significantly narrows down the complexity of the synthesis problem by systematically eliminating unsuitable technologies from consideration. The major shortcoming of the RenewIslands method is that the user is required to define the synthesis alternatives using heuristics, i.e. all decisions on structure, sizing and operation need to be specified manually. In general, the optimal solution is not included within this limited number of alternatives and the RenewIslands method will thus lead to suboptimal solutions only.

Voll et al. [25] successfully developed a method for the automated synthesis of distributed energy supply systems. It is implemented as "eSynthesis" module into the TOP-Energy framework $[29,30]$. The key concept is to apply rigorous, superstructure-based optimization to the configuration, sizing and operation of energy systems. To circumvent the manual definition of a superstructure containing all possible synthesis alternatives, a successive optimization approach is realized that automatically generates, optimizes and expands a set of superstructure models until the optimal solution is found. For this purpose, the method includes an algorithm for automated superstructure and model generation which only needs a set of specified technologies as input. This algorithm makes use of the P-Graph based maximal structure generation method [31]. The (initially) generated superstructure model is successively optimized and expanded until it yields the optimal solution. While the method has been shown to allow for efficient synthesis of decentralized energy supply systems [25], the technologies considered in the superstructure should be limited to meaningful options. Otherwise, excessively large superstructures lead to increased computational effort which may even become prohibitive.

To enable the efficient synthesis of renewable energy systems, the two discussed approaches have been integrated as follows (Fig. 2): In a first step, the RenewIslands method is used to reduce the complexity of the considered synthesis problem by preselecting promising candidate technologies. Next, instead of assessing the identified technologies in scenario-type simulation studies $[11,26]$, they are fed into the superstructure-based optimization framework to determine the optimal renewable energy system.

In the authors' opinion, the proposed hybrid approach has the potential to combine the benefits of heuristic- and optimizationbased synthesis. First of all, RenewIslands provides a transparent method with clearly defined rules for the preselection of candidate technologies. This avoids the use of subjective assumptions as often required in current practice. Furthermore, the heuristic preselection of candidate technologies leads to a significant complexity reduction and facilitates optimization-based synthesis: Most importantly, the superstructure is limited to contain only the essential equipment options. Consequently, the modeling of excluded equipment options can be omitted which is often the most time-consuming step in practice. Algorithmically, all equations and

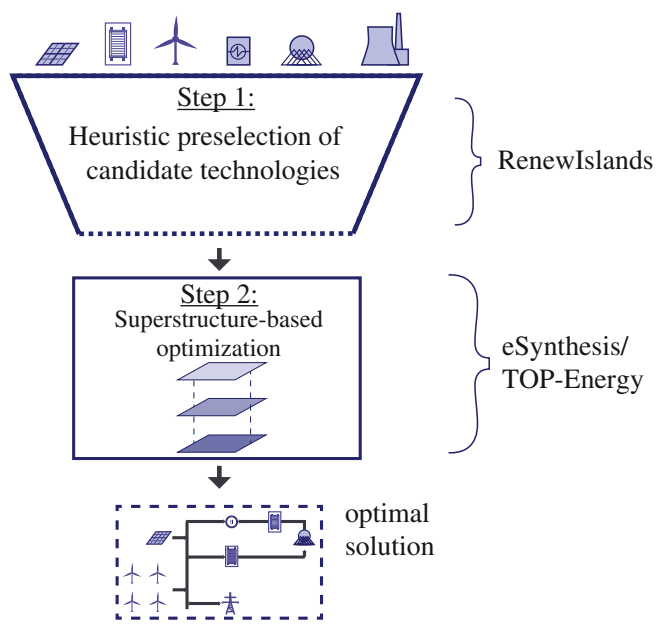

Fig. 2. Proposed two-step hybrid approach for the synthesis of renewable energy systems. The candidate technologies identified by heuristic preselection (step 1) are employed in superstructure-based optimization (step 2) to determine the optimal renewable energy system. 
(possibly binary) variables employed for the description of the structure, sizing and operational behavior of the excluded equipment can be dropped for every time-step within the considered time horizon. For instance, in an hourly analysis of a full year, i.e. $8760 \mathrm{~h}$, the omission of only one constraint for the operational behavior (which needs to be defined in every hour) reduces the optimization model already by 8760 equations. Accordingly, substantial benefits in the solution process and a significantly reduced computational effort are expected. Besides, the usually time-consuming effort for data collection and parameterization becomes obsolete. In summary, the proposed hybrid approach allows employing superstructure-based optimization to determine the optimal synthesis solution for complex real-world problems and it is not necessary to resort to scenario-type simulation studies that consider only a very limited set of synthesis alternatives.

\section{Case study "Mljet Island"}

In the following, a real world case study - the Island of Mljet, Croatia - is considered. The island of Mljet is located on the Eastern part of the Adriatic Sea. Mljet measures $37 \mathrm{~km}$ in length by $3.2 \mathrm{~km}$ average width and an area of $100 \mathrm{~km}^{2}$. General population of Mljet from the 2001 census was 1111 inhabitants. Local economy mainly relies on viticulture, olive growing and tourism. This case study has already been analyzed by Krajačić et al. [26] with the original form of the RenewIslands method, i.e. using scenario-type simulation studies instead of rigorous optimization. The objective was set to identify energy supply systems for Mljet that maximize the use of locally available renewable resources and to investigate their economic viability. In the present work, the proposed hybrid approach is applied to the same objective and the results are compared to the original study [26].

The case of Mljet Island is a typical example for renewable energy systems for which the balance between installed generation and storage capacities is crucial [32-34]. Balancing generation and storage is particularly complex since it does not only affect all three hierarchically dependent synthesis levels (Fig. 1) but also because both generation and demand are fluctuating and coupled for all time steps via the storage system. This additional interdependency needs to be adequately addressed within the synthesis of the renewable energy system.

\subsection{Preselection of candidate technologies}

Following the proposed hybrid approach, in a first step, candidate technologies for a renewable-based energy system for Mljet Island are determined by heuristic preselection. According to the

Table 1

Needs and resources of Mljet Island, qualitatively assessed according to the RenewIslands method [24,26].

\begin{tabular}{lll}
\hline & Level & Geographic distribution \\
\hline Needs & & \\
Electricity & Medium & Dispersed \\
Heat & Low & Dispersed \\
Cold & Low & Dispersed \\
Resources & & \\
Wind & Medium & - \\
Solar & Medium & - \\
Hydro & Medium & - \\
Biomass & High (but protected) & - \\
Geothermal & Low & - \\
Grid connection & Strong & - \\
Natural gas pipeline & No & - \\
LNG terminal & No & - \\
Oil terminal/refinery & No & - \\
Oil derivatives terminal & No & - \\
\hline
\end{tabular}

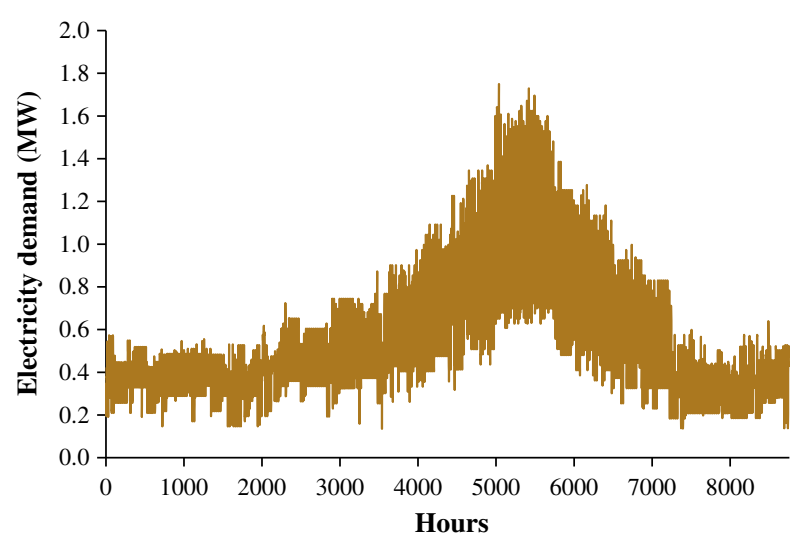

Fig. 3. Electricity demand of Mljet Island in 2010, calculated from available measurement data of the year 2002 (hourly average, assumed increase of 7\% p.a.).

RenewIslands method, starting point for the preselection is a systematic acquisition and qualitative assessment of the local needs and available resources (Table 1). Mljet is connected to the mainland with two undersea electricity grid connections. There is no electricity generation capacity on the island. Due to a lack of potable water in summer, three desalination plants are installed on the island. Together with a 300-bed hotel, these desalination plants represent the largest electricity consumers. An analysis of measurement data from the year 2002 and extrapolation to the year 2010 yields a total demand of approx. $4.6 \mathrm{GWh} / \mathrm{a}$ with a peak load of roughly $1.8 \mathrm{MW}$ (Fig. 3). The electricity demand is therefore assessed as "medium". The demand for heating and cooling is assessed as "low" because the climate of Mljet is Mediterranean with average yearly temperatures in the range of $9^{\circ} \mathrm{C}$ in January to $24{ }^{\circ} \mathrm{C}$ in July. As renewable resources, mainly wind and solar energy are available on the island of Mljet. Based on measurement data for solar irradiation and wind speed, both are assessed as "medium". Biomass is abundantly available in form of Aleppo pine. However, it is not usable because it is located in a natural reserve. There are no facilities for the import or processing of fossil fuels or its derivatives available on the island. Transport fuel is delivered via ship and distributed via one fuel station for the entire island. This assessment of local needs has been adopted from the original publication [26]. More detailed information is also given in [24].

Based on this assessment, the heuristic if-then-relations provided by the RenewIslands method are applied for equipment preselection. In total, 58 rules are defined in the RenewIslands method of which 34 apply to the present case study. Their application yields that 14 of 17 conversion technologies and 5 of 7 storage technologies can be eliminated from the general set of technologies defined in the RenewIslands method (Fig. 4). For example, geothermal plants are excluded following the rule that geothermal plants are only considered if the resource is classified as "high". The preselection reduces the number of equipment considered from 24 to only 5. In particular, the provision of heat and cold can be excluded from further consideration due to the low demand for these needs and their dispersed geographic distribution. Hence, the synthesis task reduces to a renewable electricity supply system. Apart from the existing mainland grid connection, the remaining candidate technologies are wind turbines, photovoltaic panels and a hydrogen loop consisting of an electrolyser, a fuel cell and hydrogen storage. Further details on the preselection are provided in [24].

\subsection{Superstructure-based optimization}

The preselection step has identified five candidate technologies for a renewable electricity supply system for Mljet Island. Now, in 


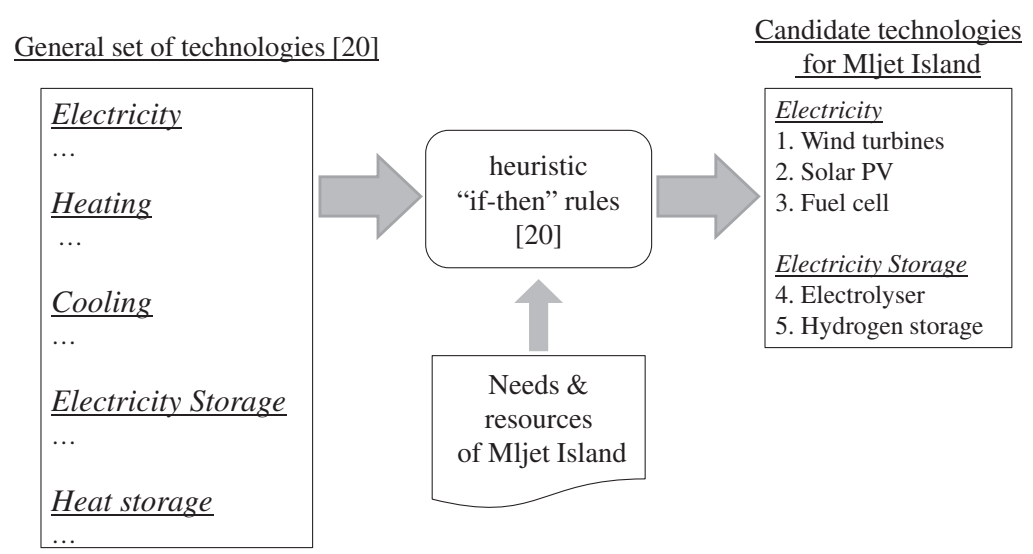

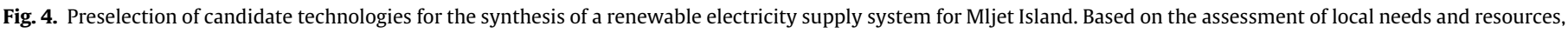
heuristic "if-then" rules of the RenewIslands method are used to eliminate unsuitable options from the general set of technologies.

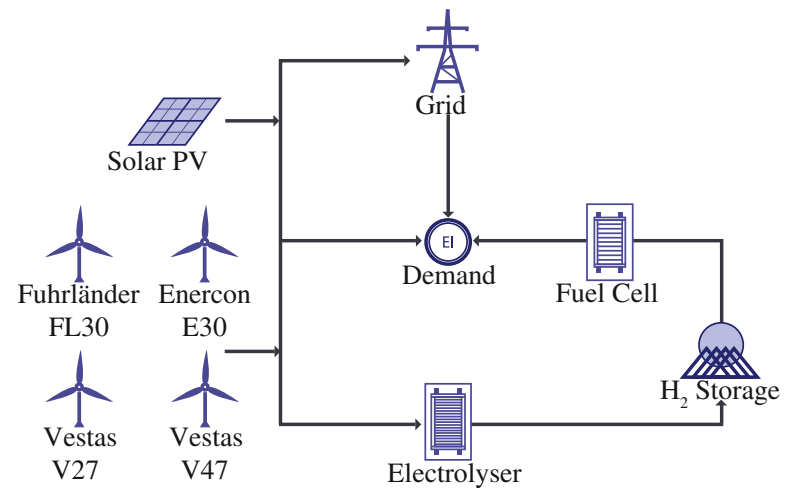

Fig. 5. General superstructure of a renewable electricity supply system for Mljet Island based on the preselected technologies.

the second step of the proposed hybrid method, the optimal system is determined by superstructure-based optimization considering only these candidate technologies.

The method developed by Voll et al. [25] provides an algorithm for automated superstructure and model generation. Its application to the candidate technologies yields the general superstructure illustrated in Fig. 5. The renewable electricity is produced by different types of wind turbines and photovoltaic panels. The produced electricity can be used to satisfy the local demand, to operate the electrolyser charging the hydrogen storage or it can be exported to the mainland. Demand satisfaction is also possible by operating the fuel cell discharging the hydrogen storage or by importing electricity from the mainland.

Table 2

Cost and performance parameters for the equipment considered in the general superstructure [26].

\begin{tabular}{llll}
\hline Equipment & $\begin{array}{l}\text { Nominal power } \\
(\mathrm{kW})\end{array}$ & Efficiency & $\begin{array}{l}\text { Investment costs } \\
(\text { EUR/kW })\end{array}$ \\
\hline Fuhrländer FL 30 & 33 & Power curve & 1500 \\
Enercon E-30 & 300.5 & Power curve & 1500 \\
Vestas V27 & 225 & Power curve & 1500 \\
Vestas V47 & 660 & Power curve & 1500 \\
PV & Continuous & 0.058 & 5500 \\
Fuel cell & Continuous & 0.5 & 3000 \\
Electrolyser & Continuous & 0.6 & 2700 \\
H2 Storage & Continuous & - & $13^{\mathrm{a}}$ \\
\hline
\end{tabular}

\footnotetext{
a EUR/kWh
}

The underlying technology models are identical to the original models [26], i.e., exactly the same data for demand, operating behavior, costs, etc. is used (Table 2). This implies the following assumptions:

- all calculations are based on known annual time series with discrete time steps of $1 \mathrm{~h}$ for demand and generation data (wind speeds, solar irradiation, etc.; e.g. Fig. 3);

- different types of wind turbines are available in discrete equipment sizes only and part-load behavior is modeled with the help of power curves:

- for all other technologies, constant efficiencies are assumed and neither part-load behavior, minimum part-load restrictions or minimum technology sizes are considered;

- the specific investment costs of the equipment are independent from equipment sizing, i.e. no economy of scale effects are modeled;

- the share of renewable electricity in the grid is not limited, i.e., $100 \%$ demand satisfaction by renewable resources is allowed; however, the export of excess electricity is limited to $30 \%$ of the annual renewable production;

- the hydrogen loop can only be operated by renewable resources.

It should be emphasized that the presented framework does not require these assumptions but can accommodate more general systems. The assumptions have been deliberately introduced to reproduce the results of the previous study [26].

With these assumptions, the optimization model is formulated as mixed-integer linear program (MILP) as follows:

$$
\min _{\underline{x} \underline{\underline{y}} \underline{\underline{y}}} z=\underline{a}^{T} \underline{x}+\underline{c}^{T} \underline{y}
$$

$$
\begin{array}{ll}
\text { s.t. } & A \underline{x}+B \underline{y}=0 \\
& C \underline{x}+D \underline{y} \leqslant \underline{b}
\end{array}
$$

where $\underline{a}, \underline{x} \in \mathbb{R}^{n}, \quad y \in \mathbb{Z}^{k}$,

$$
\begin{array}{ll}
\underline{c} \in \mathbb{R}^{k}, & \underline{b} \in \mathbb{R}^{m}, \\
A \in \mathbb{R}^{g \times n}, & B \in \mathbb{R}^{g \times k}, \\
C \in \mathbb{R}^{m \times n}, & D \in \mathbb{R}^{m \times k}
\end{array}
$$

The function $z(\underline{x}, \underline{y})$ is the objective function for the synthesis and is further specified below. The optimization is subject to constraints that represent the mathematical description of all 
Table 3

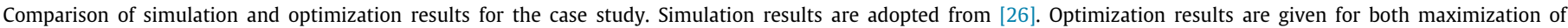

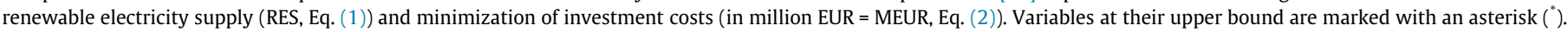

\begin{tabular}{|c|c|c|c|c|c|c|c|c|}
\hline Type & $\begin{array}{l}\text { Wind } \\
\text { (MW) }\end{array}$ & $\begin{array}{l}\text { PV } \\
(\mathrm{MW})\end{array}$ & $\begin{array}{l}\text { Electrolyser } \\
(\mathrm{MW})\end{array}$ & $\begin{array}{l}\text { Fuel cell } \\
(\mathrm{MW})\end{array}$ & $\begin{array}{l}\mathrm{H}_{2} \text { storage } \\
(\mathrm{MWh})\end{array}$ & $\begin{array}{l}\text { Renewable } \\
\text { resources } \\
(\%)\end{array}$ & $\begin{array}{l}\text { Investment costs } \\
\text { (MEUR) }\end{array}$ & $\begin{array}{l}\text { Solution time } \\
\text { (s) }\end{array}$ \\
\hline Simulation & 1.2 & 7.8 & 4 & 1.8 & 188 & 100 & 63.4 & - \\
\hline Max.\% RES & 2.1 & 8.5 & 7.9 & 1.8 & $5000^{*}$ & 100 & 141.2 & 54 \\
\hline Min. investment & 5.3 & & 2.5 & 1.8 & 487 & 100 & 26.3 & 105 \\
\hline
\end{tabular}

given data and information on the synthesis problem. For instance, the equality constraints $A \underline{x}+B \underline{y}=0$ include the energy balances which are defined for every hour of the year; the inequality constraints $C \underline{x}+D y \leqslant \underline{b}$ model e.g. operational limits, such as the export of excess electricity. The vector $\underline{x}$ contains all continuous variables, e.g. for modeling the size and power supply of the photovoltaic panels. The vector $y$ on the other hand comprises integer variables, which are only necessary for the sizing of the wind turbines in the present case study. Finally, the matrices $A, B, C$ and $D$ and the vectors $\underline{a}$ and $\underline{c}$ capture all constant coefficients representing the input data (time series, efficiencies, costs, etc.). Formulated that way, the problem can be solved to the global optimal solution in a single run using CPLEX $^{\circledR} 12.5$ as solver on a $3.3 \mathrm{GHz}$ Intel ${ }^{\circledR}$ Core $^{\mathrm{TM}} \mathrm{i}-5-2500 \mathrm{CPU}$ with 3.23 GB RAM.

\subsubsection{Maximization of renewable electricity supply}

In accordance to the original study [26], optimization is performed aiming at a maximum share of renewable energies. Hence, minimization of electricity import from the grid $\left(x_{t}^{\text {grid-demand }}\right)$ is used as objective function (the index $t$ refers to each time step).

$\min z=\sum_{t=1}^{8760} x_{t}^{\text {grid-demand }}$

The problem is solved to its optimal solution in less than a minute (cf. Table 3). The solution comprises all information on the structure of the energy system, the sizing of the technologies and a schedule for operation in every hour of the year (i.e. 8760 time steps). Since the objective function addresses only the minimization of electricity import, cost trade-offs are not taken into account. Instead, the optimization selects candidate technologies according to their provision of renewable energy. Hence, for a share of $100 \%$ renewable electricity, a solution is found that proposes large installations of photovoltaic panels and a huge storage capacity. The optimized storage capacity takes the value of its upper bound. For such large storage capacities, infinitely many solutions exist that ensure a $100 \%$ renewable electricity supply, i.e. this solution is non-unique. Moreover, the proposed solution leads to large investment costs of 141.2 million EUR (million EUR = MEUR, Table 3) - typically a serious obstacle for realization in practice.

\subsubsection{Minimization of investment costs}

To avoid the economically undesirable oversizing of equipment, the optimization is repeated using the minimization of investment costs as objective function. Hence, cost trade-offs are now explicitly incorporated within the synthesis problem via the objective function:

$\min z=\sum_{l} a_{l}^{\text {inv }} x_{l}^{\text {size }}+\sum_{k} c_{k}^{\text {inv }} y_{k}$

The coefficients $a_{l}^{\text {inv }}$ and $c_{k}^{\text {inv }}$ denote the specific investment costs of the continuously sized equipment $x_{l}^{\text {size }}(l=$ photovoltaic panels, electrolyser, etc.) and the discretely sized equipment $y_{k}$ ( $k=$ different types of wind turbines), respectively. For this objective function, a constraint is added assuring that the share of renewable resources must be $100 \%$. Again the optimal solution is found within an acceptably short solution time of less than 2 min. Compared to the prior solution, an investment costs reduction of more than $80 \%$ is achieved (26.3 MEUR instead of 141.2 MEUR). Moreover, the optimization-based solution is also about $59 \%$ cheaper than the solution derived in the original simulation study (63.4 MEUR). The optimization-based synthesis solution (Fig. 6) represents the cheapest concept to supply Mljet Island with $100 \%$ electricity from locally available renewable resources with the technologies considered. The corresponding optimized schedules for operation of the wind turbines and the hydrogen storage are shown in Fig. 7. From the set of candidate technologies identified by preselection, photovoltaic panels are not included in the

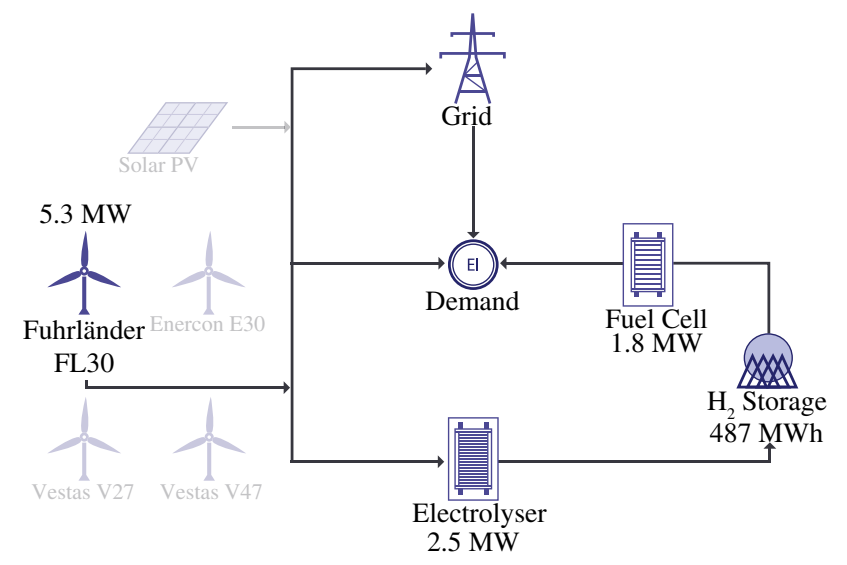

Fig. 6. Minimum investment costs solution of a $100 \%$ renewable electricity supply system for Mljet Island, identified by optimization of the general superstructure. Units not selected are shown in pale nuance.

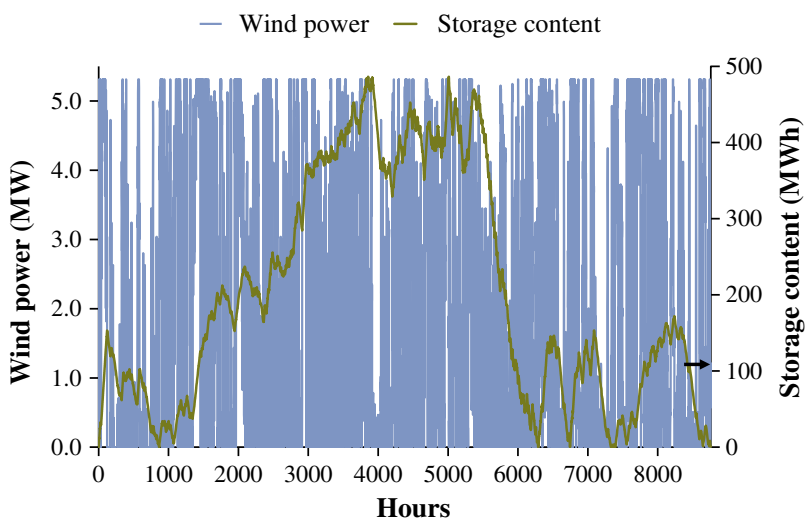

Fig. 7. Hourly production of the wind turbines installed in the minimum investment costs solution and optimal schedule for the storage level, identified by optimization of the general superstructure. 
minimum investment costs solution. For the assumed specific investment costs, efficiency and given solar irradiation (Table 2), the photovoltaic panels are too expensive or less cost-effective, respectively, compared to wind power. Remarkably, the same holds for all other types of wind turbines than "Fuhrländer FL30", although the specific investment costs of all wind turbine types are the same. This demonstrates that the optimization balances both cost and performance trade-offs between the candidate technologies with respect to structure, sizing and operation for the given data. It is important to emphasize that these insights can only be attained as an output of the optimization (and not already within the preselection step). With respect to the balance of installed generation and storage capacities, the solution identifies the following configuration to be cost optimal: The installed generation capacity of 5.3 MW wind power is almost three times higher than the peak load of $1.8 \mathrm{MW}$ and produces roughly four times more energy than the annual demand of the island. Yet, due to the temporal offset between generation and demand, only $38 \%$ of the island's energy demand can be covered directly from the wind turbines. Therefore, the excess energy is mainly used to operate the hydrogen storage, which is optimally sized to a capacity of $487 \mathrm{MWh}$ ( $\sim 11 \%$ of the annual demand). To ensure that the excess energy can be stored and used when needed, the storage can be charged with up to $2.5 \mathrm{MW}$ by the electrolyser and discharged with up to $1.8 \mathrm{MW}$ by the fuel cell. However, the hydrogen loop possesses a low round-trip efficiency of only $30 \%$.

\subsection{Case study conclusions}

This case study demonstrates the applicability of the proposed hybrid approach for the synthesis of renewable energy systems. The heuristic preselection step efficiently narrows down the problem complexity: In the studied example, five essential candidate technologies are chosen from the initial set of 24 possible technologies. Thereby, the solution effort for the subsequent superstructure-based optimization is kept within a manageable range of 1-2 min. This is the case despite the fact that 8760 coupled time steps were simultaneously evaluated solving an MILP and no algorithmic tuning has been employed. Nevertheless, the heuristic preselection of candidate technologies does not restrict the synthesis to trivial problems. On the contrary, as discussed by analyzing different objective functions in the case study, the optimization-based synthesis balances complex cost and performance trade-offs between the candidate technologies. In consequence, the identified synthesis solution for minimum investment costs exhibits enormous savings of approximately 59\% compared to the solution derived by previous simulation [26] while retaining a $100 \%$ renewable energy supply.

\section{Evaluation of the hybrid synthesis method}

The results presented in Section 3 indicate the benefits of the proposed hybrid approach. In this section, the synthesis problem presented in the case study is analyzed in greater depth in order to further evaluate the approach with regard to its applicability for sensitivity analyses, the solution quality and the related solution effort. Additionally, the usefulness of the hybrid synthesis method is evaluated for addressing multi-criteria problems.

\subsection{Parametric studies}

In the case study, the investment costs optimal solution for a $100 \%$ renewable energy system does not include photovoltaic panels. This appears intuitive, since the assumed specific costs of photovoltaic panels are much higher than the costs for wind turbines (Table 2). However, this is certainly not always the case; e.g. the price for photovoltaic panels has dropped worldwide significantly in recent years. Within the proposed hybrid approach, the superstructure-based optimization automatically assesses all cost and performance trade-offs between the candidate technologies. Accordingly, any change in the input data, e.g. price or performance assumptions, can immediately be taken into account without the need to modify or change the optimization model. The resulting synthesis solution will again be optimal with respect to the changed input data. This feature of the proposed method can hence be used for conducting systematic analyses of crucial parameters.

To demonstrate this feature, the influence of the specific investment costs of photovoltaic panels on the optimal synthesis solution is now briefly discussed for the case study of Mljet. Similar investigations could be conducted for the costs of the wind turbines (e.g. to investigate economy of scale effects) or any other input parameter. For the sensitivity analysis, two cases are considered assuming different values of investment costs for the photovoltaic panels: Costs equal to the costs of the wind turbines (1500 EUR/kWp) and costs that make photovoltaic panels significantly cheaper than wind turbines (410 EUR/kWp). For costs of $410 \mathrm{EUR} / \mathrm{kWp}$ the original cost ratio between wind turbines and photovoltaic panels is exactly inverted. The corresponding optimal synthesis solutions for minimization of investment costs are presented in Table 4.

For equal investment costs, photovoltaic panels now contribute substantially to the installed generation capacity $(2.8 \mathrm{MW}$ or almost $50 \%$ ). As a major result of this mix of renewable generators, both the electrolyser size and the storage capacity can be decreased. However, while the electrolyser size can be decreased by $36 \%$, the storage capacity can only be reduced by $14 \%$. In total, the necessary investment costs are reduced by $10 \%$.

When photovoltaic panels become much cheaper than wind turbines, naturally the installed capacity of photovoltaic panels rises further in the optimal solution (5.3 MW). However, a complete substitution of wind generation capacities by photovoltaic - as has been the case between wind and photovoltaic panels for the inverse price ratio - is not observed. Hence, a complete substitution is not economically favorable. Instead, wind turbines still should contribute with 2 MW (more than 25\%) to the total installed generation capacity. While the storage capacity can be decreased by another $9 \%$, the electrolyser size remains almost the same as in the previous case. The total investment costs are reduced by another $17 \%$.

Table 4

Installed equipment capacities in the optimal synthesis solutions for varying prices of photovoltaic panels and minimization of investment costs as objective function.

\begin{tabular}{|c|c|c|c|c|c|c|c|c|}
\hline $\begin{array}{l}\text { PV invest. costs } \\
(\text { EUR/kWp) }\end{array}$ & $\begin{array}{l}\text { Wind } \\
\text { (MW) }\end{array}$ & $\begin{array}{l}\text { PV } \\
(\mathrm{MW})\end{array}$ & $\begin{array}{l}\text { Electrolyser } \\
(\mathrm{MW})\end{array}$ & $\begin{array}{l}\text { Fuel cell } \\
(\mathrm{MW})\end{array}$ & $\begin{array}{l}\mathrm{H}_{2} \text { storage } \\
(\mathrm{MWh})\end{array}$ & $\begin{array}{l}\text { Renewable } \\
\text { resources } \\
(\%)\end{array}$ & $\begin{array}{l}\text { Investment costs } \\
\text { (MEUR) }\end{array}$ & $\begin{array}{l}\text { Solution time } \\
\text { (s) }\end{array}$ \\
\hline 5500 & 5.3 & & 2.5 & 1.8 & 487 & 100 & 26.3 & 105 \\
\hline 1500 & 3 & 2.8 & 1.6 & 1.8 & 421 & 100 & 23.7 & 88 \\
\hline 410 & 2 & 5.3 & 1.5 & 1.8 & 385 & 100 & 19.7 & 110 \\
\hline
\end{tabular}


Table 5

Definition of scenarios for the case study by allowing different subsets of technologies [26]. ${ }^{\text {a }}$ Scenario 12 represents the general superstructure analyzed in Section 3.

\begin{tabular}{|c|c|c|c|c|c|}
\hline Original scenario number [26] & Wind & PV & Electrolyser & Fuel Cell & H2 Storage \\
\hline 2 & $\nu$ & & & & \\
\hline 4 & & レ & & & \\
\hline 6 & 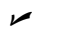 & $\boldsymbol{\nu}$ & & & \\
\hline 8 & $\nu$ & & レ & $\nu$ & $\nu$ \\
\hline 10 & & $\boldsymbol{V}$ & $\boldsymbol{\nu}$ & $\nu$ & レ \\
\hline 12 (general) & $\boldsymbol{\nu}$ & $\boldsymbol{r}$ & $\boldsymbol{\nu}$ & レ & $\nu$ \\
\hline
\end{tabular}

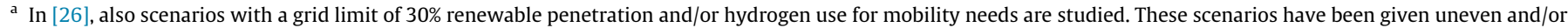
higher numbers in $\underline{\underline{[26]}}$ and are not considered in the present work.

This brief analysis demonstrates that the proposed synthesis method can readily be applied to perform systematic studies on the influence of input parameters on the optimal solution.

\subsection{Evaluation of the solution quality}

The superstructure used in the case study (Fig. 5) is the most general superstructure considered. It contains the five preselected candidate technologies and encodes all theoretically possible alternatives for their combination. In [26], additional scenarios are analyzed that explicitly consider other subsets of the candidate technologies (Table 5). It is instructive to consider those scenarios as well. The number of considered candidate technologies increases over the given scenarios and that for each scenario a synthesis solution exists derived by simulation. These solutions are used as benchmark for the assessment of the solution quality of the optimization-based synthesis. Additionally, the influence of the number of candidate technologies on the solution effort is analyzed (Section 4.3).

In accordance to the prior investigation conducted in the case study, the superstructures associated with each scenario are optimized with respect to two different objective functions: Minimization of electricity import (i.e. maximization of the share of renewable resources) and minimization of investment costs. The results are shown in Fig. 8.

For an objective function maximizing the share of renewable resources, optimization-based synthesis increases the share of renewable resources for scenarios 2 and 6 by $8 \%$ and 3\%, respectively. Furthermore, optimization confirms that a share of $35 \%$ is the maximum value to be reached when only photovoltaic panels are installed (scenario 4). Naturally, no improvements can be found for scenarios 8,10 and 12 with a share of renewable resources already at its maximum level of $100 \%$. However, as shown in Fig. 8 b), the optimization-based synthesis solutions require less investment costs. The cost reductions range between $11 \%$ (scenario 10) and $59 \%$ in the general superstructure (scenario 12, cf. Section 3). Even for scenario 8, which does not employ expensive photovoltaic pan- els and is therefore structurally close to the optimal solution, the savings are still higher than $30 \%$. In a nutshell, the optimizationbased synthesis solutions are always better or equal to the results from the simulation studies [26], as should be expected from a rigorous MILP approach. The most significant improvements appear in the scenarios with the highest degrees of freedom (scenarios $8-12$, with energy storage), affirming the benefit of superstructure-based optimization in dealing with the complex trade-offs between structure, sizing and operation of the technologies.

\subsection{Evaluation of the solution effort}

The previous comparison points out the strength of superstructure-based optimization in finding optimal solutions for various synthesis problems. Still, in practice, it is crucial to keep the required solution effort manageable for a successful application of the superstructure-based optimization step within the proposed hybrid approach. Due to the hierarchic nature of the synthesis problem (cf. Section 1), the number of preselected candidate technologies substantially affects the solution effort.

For the scenarios introduced in Section 4.2, the solution effort for minimizing investment costs is illustrated in Fig. 9. The solution time increases exponentially with an increasing number of candidate technologies in the scenarios. While e.g. the scenarios with only one technology (scenarios 2,4 ) require roughly $2 \mathrm{~s}$ to be solved, the general superstructure with five candidate technologies takes already more than 100 s, i.e. more than 50 times longer. For that reason, the necessity to limit the number of candidate technologies becomes central. In the proposed hybrid approach, the number of candidate technologies is bounded by the preselection step utilizing the RenewIslands method. It should be emphasized that the preselection step is independent from the employed equipment models. In the present case study, simple equipment models are used that mostly neglect e.g. part-load behavior. Thus, for more detailed models, the reduction in computational effort from limiting the number of candidate technologies is expected to be even more valuable.
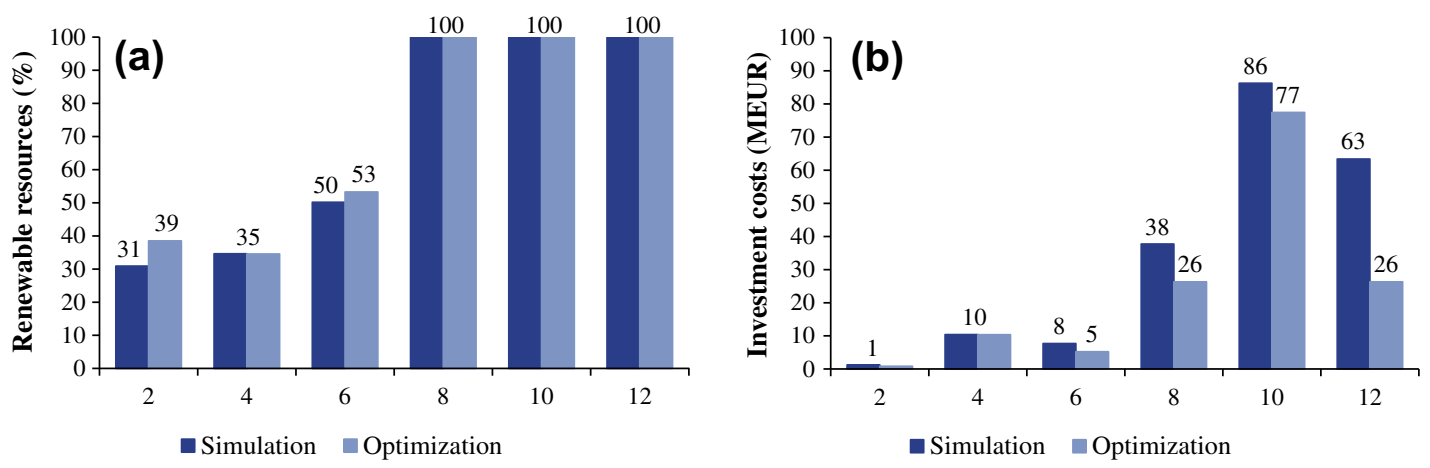

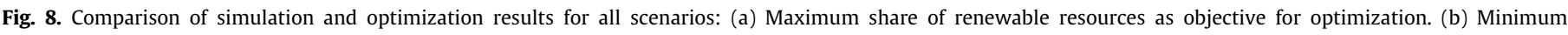
investment costs as objective for optimization, where the share of renewable resources from the simulation results is set as constraint. 


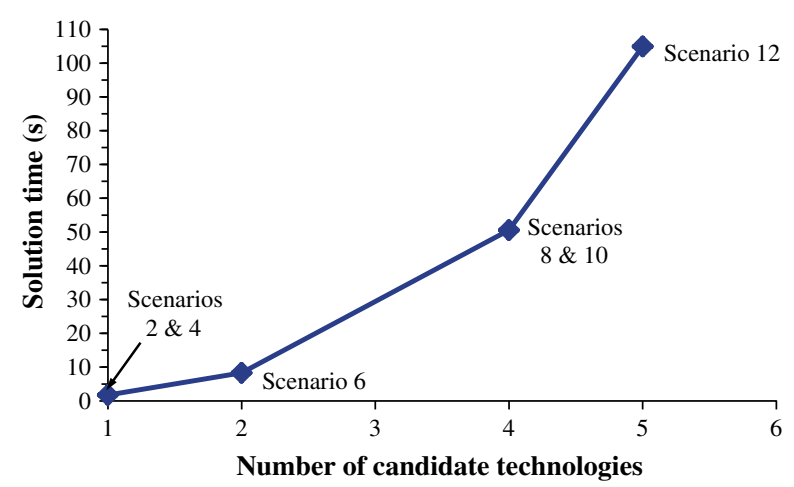

Fig. 9. Solution times for the optimization-based synthesis in dependence of the number of candidate technologies considered in the scenarios.

\subsection{Multi-objective optimization}

The reduced computational effort gained from the equipment preselection also opens up the opportunity to conduct investigations that require multiple optimization runs, e.g. multi-objective optimization [35-37]. A multi-objective analysis in turn may provide useful additional insight into the synthesis problem at hand. This feature is now illustrated for the case study. A Pareto frontier is generated using the $\varepsilon$-constraint method [38] to investigate how much investment is at least necessary for a certain share of renewable resources. The generation of the Pareto frontier requires nine additional optimization runs and is completed in $24 \mathrm{~min}$.

The slope of the Pareto frontier (Fig. 10, top) shows that the share of renewable resources can only be increased towards $100 \%$ with progressively larger costs, as progressively more equipment needs to be installed (Fig. 10, bottom). Roughly three ranges can be identified in the Pareto frontier in Fig. 10: Renewable resources supplying less than $40 \%, 40-90 \%$ or up to $100 \%$ of the
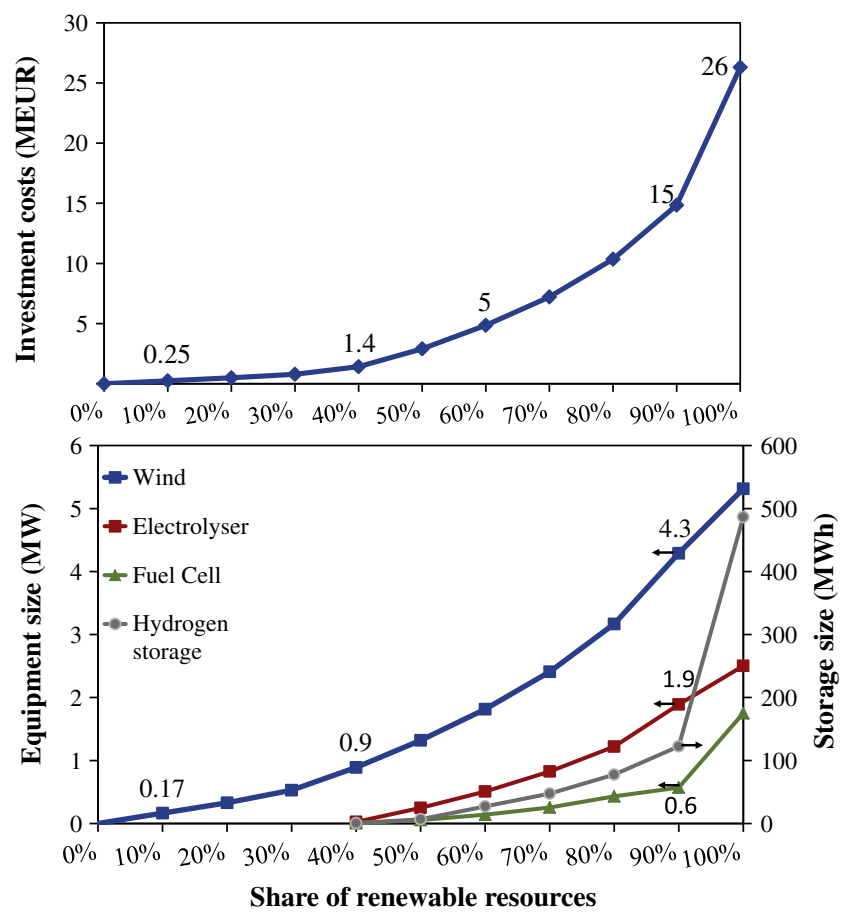

Fig. 10. Results of the multi-objective optimization for the case study. Top graph: Pareto frontier showing minimum investment costs for a given share of renewable resources. Bottom graph: corresponding equipment sizes. demand. If renewable resources supply less than $40 \%$ of the demand, it is sufficient to install wind turbines only and moderate costs of less than 1.5 MEUR occur. The wind power can be used for direct demand supply and no energy storage is needed. However, if a share of more than $40 \%$ of renewable resources is desired, it becomes favorable to compensate the temporal offset between generation and demand by providing energy storage and installing the hydrogen loop. Thus, from that point on, the costs for the electrolyser, the hydrogen storage and the fuel cell add to the total investment costs and the slope of the Pareto frontier becomes steeper. At a share of $60 \%$ of renewable resources, investment costs have reached already 5 MEUR and further increase up to 15 MEUR at $90 \%$. Due to the conversion losses that occur in the hydrogen loop, considerably more wind energy than required for demand supply needs to be harvested. Accordingly, the installed equipment size of wind turbines rises from $0.9 \mathrm{MW}$ to $4.3 \mathrm{MW}$ between $40 \%$ and $90 \%$ share of renewable resources. Likewise, the electrolyser size rises from less than $100 \mathrm{~kW}$ to almost $2 \mathrm{MW}$ to convert the harvested surplus wind power into hydrogen. Between $40 \%$ and $90 \%$, both the installed fuel cell size and the storage size can still be kept relatively low. For a share of renewable resources between $90 \%$ and $100 \%$, the slope of the Pareto frontier rises even further. In fact, the last $10 \%$ are almost equally expensive as the first $90 \%$ with investment costs increasing from 15 MEUR up to 26 MEUR. This is mostly due to fact that the fuel cell and storage capacities now need to be expanded massively; by the factors three and four, respectively, to cover the lack of wind in summer when the electricity demand is at its peak.

\section{Conclusions}

This paper presents a hybrid approach for the synthesis of renewable energy systems. The hybrid approach consists of an initial heuristic-based preselection of candidate technologies followed by a rigorous optimization. The preselection builds upon the RenewIslands method [24] and optimization is conducted superstructure-based, as developed by Voll et al. [25]. The heuristic-based preselection aims at narrowing down the inherent problem complexity induced by the variety of available renewable technologies. The superstructure-based optimization subsequently determines the optimal synthesis solution balancing all cost and performance trade-offs between the candidate technologies with respect to structure, sizing and operation for the given problem data.

The application of the hybrid approach to the case study of Mljet Island shows that the complexity of the synthesis problem can successfully be narrowed down by preselecting five promising candidate technologies from a comprehensive set of more than 20 options. For different objective functions, the implemented MILP optimization model yields the optimal solution in less than 2 min using a standard solver. Major benefits of the superstructure-based optimization are achieved when costs are taken into account. At an equal share of $100 \%$ renewable electricity, the optimization-based solution requires only $41 \%$ of the investment costs compared to a solution originally derived by simulation [26].

Further evaluations of the hybrid approach confirm its benefits. In particular, the optimization-based synthesis can conveniently be applied for systematic parametric studies on crucial inputs. Moreover, a detailed comparison of optimization results with scenariotype simulation studies [26] shows that the optimization-based synthesis is particularly valuable for the synthesis of energy systems with high degrees of freedom, e.g. systems including energy storage technologies. An analysis of the solution effort for the optimization demonstrates the fundamental role of limiting the number of candidate technologies within the preceding preselection 
step. The low computational effort achieved by the preselection in turn also enables efficient multi-criteria optimization yielding valuable insights on renewable energy systems.

Thus, the proposed hybrid approach represents an efficient method for the synthesis of renewable energy systems.

\section{Acknowledgements}

It is gratefully acknowledged that this work has been supported by the Croatian Science Foundation through the "Optimization of Renewable Electricity Generation Systems Connected in a Microgrid" collaborative research project, Grant No. HRZZ 08/40. The authors thank an anonymous reviewer for suggesting additional optimization studies in the case study.

\section{References}

[1] Lund H. Renewable energy strategies for sustainable development. Energy 2007;32(6):912-9.

[2] Mathiesen BV, Lund H, Karlsson K. 100\% Renewable energy systems, climate mitigation and economic growth. Appl Energy 2011;88(2):488-501.

[3] Edenhofer O, Madruga RP, Sokona Y, Seyboth K, Matschoss P. Renewable energy sources and climate change mitigation: special report of the intergovernmental panel on climate change. Cambridge: Cambridge Univ. Press; 2012.

[4] Frangopoulos CA, von Spakovsky MR, Sciubba E. A brief review of methods for the design and synthesis optimization of energy systems. Int J Appl Thermodyn 2002;5(4):151-60.

[5] Ibrahim H, Ilinca A, Perron J. Energy storage systems-characteristics and comparisons. Renew Sustain Energy Rev 2008;12(5):1221-50.

[6] Ramakumar R, Abouzahr I, Ashenayi K. A knowledge-based approach to the design of integrated renewable energy systems. IEEE Trans Energy Convers 1992;7(4):648-59.

[7] Elliston B, Diesendorf M, MacGill I. Simulations of scenarios with 100\% renewable electricity in the Australian National Electricity Market. Energy Policy 2012;45:606-13.

[8] Wang Y, Ronilaya F, Chen X, Roskilly AP. Modelling and simulation of a distributed power generation system with energy storage to meet dynamic household electricity demand. Appl Therm Eng 2013;50(1):523-35.

[9] Bakic V, Pezo M, Stevanovic Ž, Živkovic M, Grubor B. Dynamical simulation of PV/wind hybrid energy conversion system. Energy 2012;45(1):324-8.

[10] Beccali M, Brunone S, Cellura M, Franzitta V. Energy, economic and environmental analysis on RET-hydrogen systems in residential buildings. Renew Energy 2008;33:366-82.

[11] Segurado R, Krajačić G, Duić N, Alves L. Increasing the penetration of renewable energy resources in S. Vicente, Cape Verde. Appl Energy 2011;88(2):466-72.

[12] Chua KJ, Yang WM, Er SS, Ho CA. Sustainable energy systems for a remote island community. Appl Energy 2014;113:1752-63.

[13] Baños R, Manzano-Agugliaro F, Montoya FG, Gil C, Alcayde A, Gómez J. Optimization methods applied to renewable and sustainable energy: a review. Renew Sustain Energy Rev 2011:15(4):1753-66.

[14] Papoulias SA, Grossmann IE. A structural optimization approach in process synthesis - I: utility systems. Comput Chem Eng 1983;7(6):695-706.

[15] Bazmi AA, Zahedi G. Sustainable energy systems: role of optimization modeling techniques in power generation and supply - a review. Renew Sustain Energy Rev 2011;15(8):3480-500.

[16] Söderman J, Pettersson F. Structural and operational optimisation of distributed energy systems: process integration, modelling and optimisation for energy saving and pollution reduction - PRES 2004. Appl Therm Eng 2006;26(13):1400-8.

[17] Liu P, Gerogiorgis DI, Pistikopoulos EN. Modeling and optimization of polygeneration energy systems. Catal Today 2007;127(1-4):347-59.

[18] Ren H, Gao W. A MILP model for integrated plan and evaluation of distributed energy systems. Appl Energy 2010;87(3):1001-14.

[19] Mehleri ED, Sarimveis H, Markatos NC, Papageorgiou LG. Optimal design and operation of distributed energy systems: application to Greek residential sector. Renew Energy 2013;51:331-42.

[20] Rubio-Maya C, Uche-Marcuello J, Martínez-Gracia A, Bayod-Rújula AA. Design optimization of a polygeneration plant fuelled by natural gas and renewable energy sources. Appl Energy 2011;88(2):449-57.

[21] Bixby RE, Fenelon M, Gu Z. MIP: theory and practice - closing the gap. In: Powell MJD, Scholtes S, editors. System modelling and optimization: methods, theory, and applications. 19th IFIP TC7 conference on system modelling and optimization, July 12-16, 1999, Cambridge, UK. London: Kluwer Academic Publishers; 2000.

[22] Koch T, Achterberg T, Andersen E, Bastert O, Berthold T, Bixby R, et al. MIPLIB 2010. Math Program Comput 2011:3(2):103-63.

[23] Yuan Z, Chen B, Gani R. Applications of process synthesis: moving from conventional chemical processes towards biorefinery processes. Comput Chem Eng 2013;49:217-29.

[24] Duić N, Krajačić G, da Graça Carvalho M. RenewIslands methodology for sustainable energy and resource planning for islands. Renew Sustain Energy Rev 2008;12(4):1032-62.

[25] Voll P, Klaffke C. Hennen M, Bardow A. Automated superstructure-based synthesis and optimization of distributed energy supply systems. Energy 2013;50:374-88

[26] Krajačić G, Duić N, da Graça Carvalho M. H2RES, energy planning tool for island energy systems - the case of the island of Mljet. Int J Hydrogen Energy 2009;34(16):7015-26.

[27] Chen F, Duic N, Alves LM, da Graça Carvalho M. RenewIslands-renewable energy solutions for islands. Renew Sustain Energy Rev 2007:11(8):1888-902.

[28] Lund H, Duić N, Krajačíc G, da Graça Carvalho M. Two energy system analysis models: a comparison of methodologies and results. Energy 2007;32(6):948-54.

[29] Augenstein E, Wrobel G, Kuperjans I, Plessow M. TOP-ENERGY computational support for energy system engineering processes. In: 1st International conference from scientific computing to computational engineering (1st IC-SCCE); 2004.

[30] Voll P, Kirschbaum S, Bardow A. Evaluation of quasi-stationary simulation for the analysis of industrial energy systems. In: Favrat D, Maréchal F, editors. ECOS 2010: proceedings of the 23rd international conference on efficiency, cost, optimization, simulation, and environmental impact of energy systems. Lausanne: CreateSpace; 2010. p. 219-31.

[31] Mittelmann H. Benchmarks for optimization software. <http://plato.asu.edu/ bench.html>; 2014 [28.2.14].

[32] Vosen SR, Keller JO. Hybrid energy storage systems for stand-alone electric power systems: optimization of system performance and cost through control strategies. Int J Hydrogen Energy 1999;24(12):1139-56.

[33] Luo Y, Shi L, Tu G. Optimal sizing and control strategy of isolated grid with wind power and energy storage system. Energy Convers Manage 2014;80:407-15.

[34] Zhao B, Zhang X, Li P, Wang K, Xue M, Wang C. Optimal sizing, operating strategy and operational experience of a stand-alone microgrid on Dongfushan Island. Appl Energy 2014;113:1656-66.

[35] Miettinen K. Nonlinear multiobjective optimization. Dordrecht: Kluwer Academic Publishers; 1999.

[36] Ehrgott M. Multicriteria optimization. 2nd ed. Berlin (London): Springer; 2005.

[37] Ehrgott M, Figueira J, Greco S. Trends in multiple criteria decision analysis. New York: Springer; 2010

[38] Haimes YY, Lasdon LS, Wismer DA. On a bicriterion formulation of the problems of integrated system identification and system optimization. IEEE Trans Syst Man Cyber 1971;1(3):296-7. 